\title{
The gag proteins of human immunodeficiency virus type 1: mechanisms of virus assembly and possibilities for interference
}

\author{
Susanne Modrow ${ }^{1}$, Bernhard Kattenbeck ${ }^{1}$, Andreas von Poblotzki ${ }^{1}$, \\ Matthias Niedrig ${ }^{2}$, Ralf Wagner ${ }^{1}$, Hans Wolf ${ }^{1}$ \\ I Institut für Medizinische Mikrobiologie und Hygiene der Universität Regensburg, \\ D-93042 Regensburg, Germany \\ ${ }_{2}$ Robert-Koch-Institut, Bundesgesundheitsamt, Nordufer 40, D-13351 Berlin, Germany
}

Received: 21 June 1994

\section{Introduction}

During the last years highly developed methods in molecular biology have allowed the accumulation of detailed knowledge concerning the early steps in the course of viral infection of individual cells. This has led to the characterization of many cellular surface proteins and additional factors involved in the adsorption process of individual viruses of specific cells, e.g., for members of the retrovirus group (human immunodeficiency virus, HIV), herpes viruses (Epstein-Barr virus, herpes simplex virus), picornaviruses (poliovirus, rhinovirus, coxsackievirus), orthomyxoviruses (influenza virus) and many others. In addition to the process of virus adsorption to the surface of individual cells, much is known today about cellular factors and transactive proteins necessary for the primary steps of virus gene activation and transcription as well as about the regulated processes of gene expression and genome replication. In contrast to these early steps during virus infection and replication, only very few details are known about the late events of the virus life cycle, which lead to the release of infectious virus particles from the infected cell. One precondition for the production of these particles is the correct assembly of the individual components forming the virion structure represented by the virus capsid or core associated with the viral genome and - in the case of enveloped virus particles - the lipid bilayer of the host cell associated with viral membrane and matrix proteins. The virus structure often becomes further modified by maturation processes taking place after the diverse components have been assembled and released as premature virus particles from the infected cell, thereby finally yielding the infective virus. As with the other events occurring during the replication cycle, complex mechanisms are involved in the control and performance of this viral assembly.

After the characterization of HIV as the causative agent of the acquired immunodeficiency syndrome (AIDS) $[1,2]$ major efforts of research focused on this virus in order to understand its molecular biology as a basis for the development of 
new types of vaccines and antiviral therapies. To date this final goal has not been achieved; however, these investigations have provided a detailed knowledge of the molecular processes occurring during HIV infection. Today we know many details about the late steps in the HIV-1 life cycle, i.e., virus assembly and particle morphogenesis. This knowledge may form a basis enabling us to develop new antiviral strategies, both through vaccine design and in anti-HIV therapeutics.

\section{Formation of infectious HIV particles: a multistep procedure}

The assembly process of HIV is similar to that of other members of the lentivirus group and to that of C-type and all other retroviruses which are released from the membrane of the infected cell in the form of budding particles. Many of the individual events of this complex multistep process are not yet finally understood. The majority of the structural elements known to be required for HIV-particle formation are localized in the sequence of the precursor protein Pr55gag [3] which comprises the matrix protein $\mathrm{p} 17$, the capsid protein $\mathrm{p} 24$, the nucleocapsid protein $\mathrm{p} 7$, the protein $\mathrm{p} 6$ known as the link-protein and two smaller peptides $\mathrm{p} 2$ and p1 [46], present in infectious virions (Fig. 1). In the course of translation of the viral RNA transcripts, these proteins are synthesized in form of a polyprotein precursor (molecular mass $55000 \mathrm{Da}$ ), which is cotranslationally modified by addition of myristic acid at the penultimate amino-terminal glycine residue of the Pr55gag; the preceeding methione is removed simultaneously $[5,7]$. This myristic acid modification, as well as distinct regions of the Pr55gag itself and cellular components involved in these processes, enable the transport and incorporation of this molecule into the inner leaflet of the cell membrane [8, 9]. In addition to the cellular proteins used for myristoylation and transport of the precursor protein to the membrane, cyclophilin, a member of cellular enzymes catalyzing protein folding, has been proposed to be necessary for group-specific antigen (gag)-protein synthesis, transport and membrane targeting [10].

A further gag polyprotein representing about 5\% of the amount of Pr55gag-precursor proteins is synthesized from the same open reading frame, starting with identical sequences until a ribosomal frameshift of -1 occurs in a polyU-rich region in the unspliced mRNA in the $3^{\prime}$-region of the gag open reading frame. This event leads the translation to proceed past the normal stop codon using another reading frame, resulting in a polyprotein precursor Pr160gag-pol (Figs. 1, 2) [11, 12]. This larger polyprotein comprises the amino acids of Pr55gag up to residue 432 in the nuclear capsid protein part $\mathrm{p} 7$, which are fused to the sequences synthesized from the "pol" gene of the HIV genome (pol = open reading frame encoding virus protease, reverse transcriptase and integrase). Using this ribosomal frameshift the viral protease, reverse transcriptase and integrase are produced as parts of the resulting polyprotein precursor Pr160gag-pol. This is also myristoylated and targeted to the cytoplasmic membrane by procedures identical to those used for membrane incorporation of Pr55gag [8, 9].

Besides these two variants of gag-protein precursors, Pr55gag and Pr160gagpol, the viral envelope protein is synthesized by translation of the spliced form of viral mRNA into the endoplasmic reticulum (ER). As in cases of other transmembrane proteins, a signal peptide at the amino-terminal end of the gp160 precursor protein associates with the cellular signal recognition particle. The resulting complex is transported to the ER membrane and the viral glycoprotein is further syn- 
A)
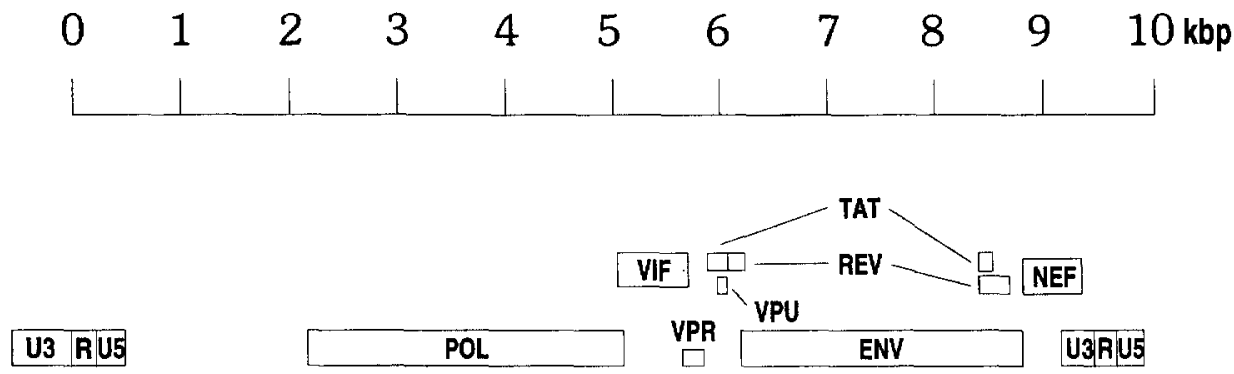

B)

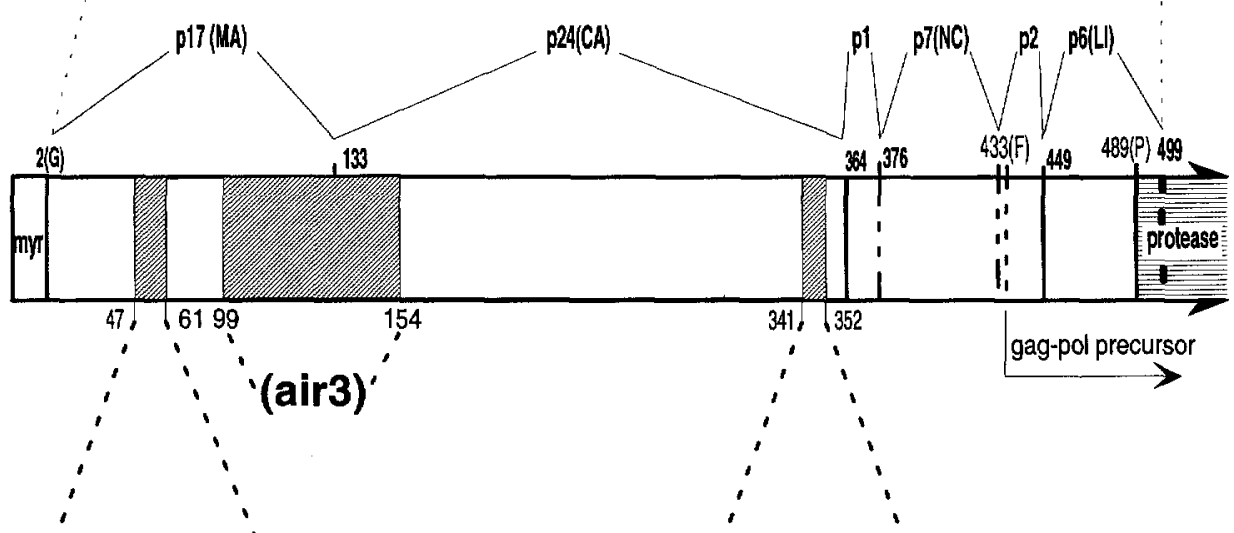

NPG LLE TSE GCRQ̈LL

ATL EEM MTA CQG

(air1)

(air2)

Fig. 1 A, B. Schematic overview of the HIV-1 genome and the proteins encoded by the diverse genome parts. A Schematic presentation of the HIV-1 genome and the regions coding for the diverse enzymatic, regulatory active and structural proteins. B Enlargement of the region coding for the Pr55gag polyprotein precursor. The regions essential for virus assembly and particle formation as mentioned in the text (airl, air2, air3) and also the cleavage sites of the HIV-specific protease and the resulting protein parts are indicated. The site where the protein sequences encoded by the pol-open reading frame is fused to the Pr55gag protein due to ribosomal frameshifting is indicated

thesized as described for transmembrane proteins type 1 [13]. In passing the Golgi apparatus, several amino acids of gp 160 are modified by glycosylation and the protein is transported and incorporated into the cellular membrane $[14,15]$. During this process the gp 160 precursor protein is endoproteolytically cleaved by a cellular furin protease into the external glycoprotein gp120 necessary for interaction with CD4-receptor proteins at the surface of T helper cells and into the transmem- 
brane protein gp41 [7, 16-18]. Both proteins remain associated by a noncovalent linkage.

By these two processes - targeting the myristoylated forms of Pr55gag and Prl60gag-pol via a cytoplasmic pathway to the plasma membrane and use of the general transport pathway for transmembrane and secretory proteins for targeting of the HIV transmembrane components gp120/gp41 - all structural and enzymatically active components of the virus become associated with the cytoplasmic membrane of the cell (Fig. 2 A). During the following steps of HIV assembly two events have to occur: first, the viral mRNA genome must interact via its $\varphi$-site, which is located upstream of the start of the open reading frame coding for the gag protein and is present only in complete and unspliced messengers, with the RNA-binding domain in the nucleocapsid protein portion of the Pr55gag polyprotein [19]. This region shows similarities to nucleic acid binding zink-finger motifs [20-23]. Second, the individual protein molecules - Pr55gag, Pr160gag-pol, and gp120/gp41 - interact to form clusters with high concentrations of the indicated proteins at the lipid bilayer $[24,25]$. They can be identified electron microscopically as electrondense regions underneath the cytoplasmic membrane $[4,26,27]$. These regions represent the initial budding structures. Membrane protrusions develop, which proceed to form rounded, spherical membraneous particles containing two copies each of the HIV-RNA genome (Fig. 2 B, C). These structures are finally released from the surface of the cell and represent non-infective, premature viral particles. The role of the accessory proteins vpr, vpu and vif in these late steps of the budding process remains to be elucidated $[28-31]$.

The majority of the maturation steps occur after the premature HIV particles have been released, whereby an important role is performed by the viral protease. The sequence of this enzyme is localized in the Pr160gag-pol-precursor protein immediately downstream of the frameshift site, i.e., the protease sequences are directly fused to the gag protein region. The HIV protease is a member of aspartate proteases and has to dimerize to yield an active enzyme [32-34]. Furthermore, the enzymatic activity of the protease is dependent on an acidic milieu, present only inside the released particles [35]. These two preconditions for HIV protease activity - dimerization and an acidic environment - prevent proteolytic cleavage of cellular proteins. Nevertheless, there are some recent indications that a partial intracellular activation of the protease may contribute to the cytotoxic effects of the virus (von der Helm, unpublished observation and [36]). Optimal enzyme activity is, however, only present in the released particles, where the high density of Pr55gag and Pr160gag-pol proteins promote dimerization of the protease moieties in the precursor molecules and the enzyme is excised by autocatalytical activation [37]. By the endoproteolytic activity of the HIV protease the Pr55gag- and Pr160gag-pol-precursor molecules are cleaved into the proteins identified in infectious viral particles as p17MA, p24CA, p7NC, p6Li and the previously mentioned viral enzymes $[6,7,38,39]$. The matrix protein $\mathrm{p} 17 \mathrm{MA}$ remains associated with the viral membrane mainly by the myristic acid modification. p24CA is arranged in a way to give the conical core structure found only in mature HIV particles [40]; p7NC is complexed by the zink-finger domain with the RNA genomes and p6LI seems to form the core-envelope link, facilitating the closure of the spherical core (Fig. 2D) [30, 41, 42]. Since all these steps during the assembly process are highly critical for the formation of infectious HIV particles, each one may be individually targeted to disturb and destroy the infectivity of the virus. Therefore, much work has been done to define the molecular details of the individual 

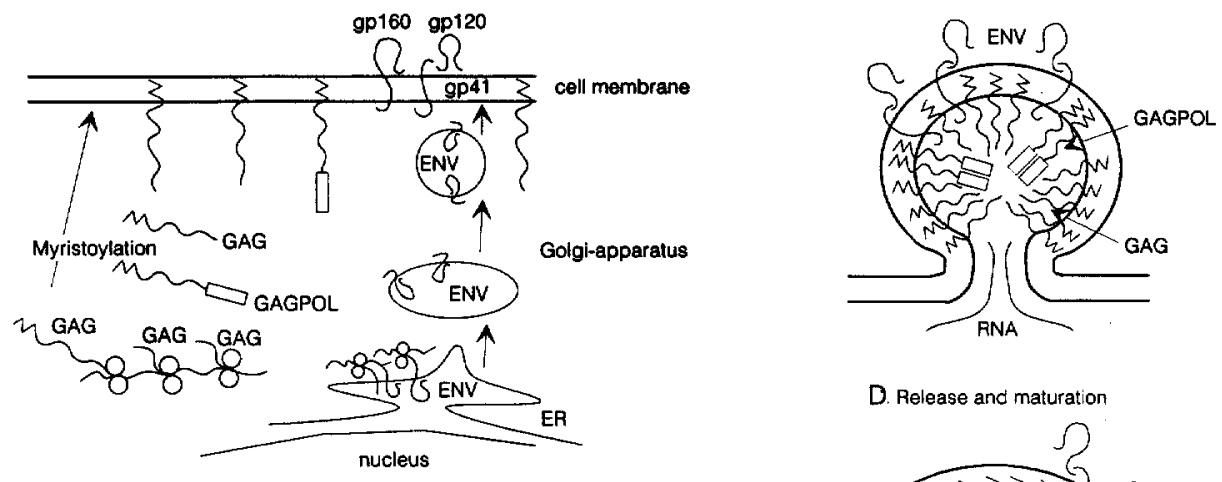

D. Release and maturation

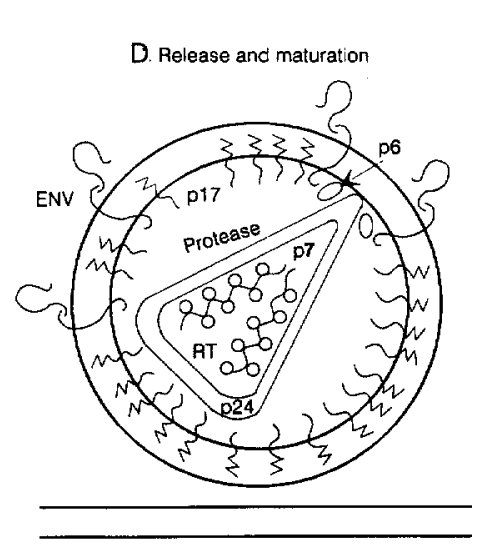

B. Interaction between protein precursors

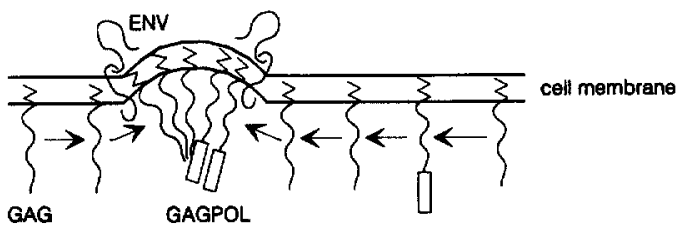

Fig. 2 A -D. Presentation of the individual steps during HIV-assembly and particle formation A Pr55gag and Pr160gag-pol precursor proteins are translated from genomic mRNA in the cytoplasma of the cell, myristoylated, transported to and incorporated into the cell membrane. Spliced forms of mRNA encoding the sequences for the envelope proteins are translated into the endoplasmic reticulum (ER), modified by glycosylation and transported via the Golgi apparatus to the cell membrane. During this process the precursor protein gp 160 is processed into gp 120 and gp41 by the cellular protease furin. B The membrane associated Pr55gag and Pr160gag-pol polyprotein precursor interact to form a submembraneous layer of high density of these proteins; the complexes of the envelope proteins become incorporated into these patches containing high concentrations of gag-proteins. $\mathbf{C}$ The membrane regions containing high concentrations of viral structural proteins begin to form protrusions representing the initial budding structures. During this process two genomic RNA molecules become associated via their $\varphi$ sequences with the zinkfinger domains located within the $\mathrm{p} 7 \mathrm{NC}$ protein part of the Pr55gag polyprotein precursor. The budding complex further rounds up to form a spherical particle still associated with the surface of the cell. D The immature HIV particle is finally released from the cell surface. Due to the high concentration of structural proteins the protease domains dimerize as parts of the Pr160gag-pol polyprotein precursor molecules thus forming the active protease which is cleaved from the precursor by autocatalytical activation. The active protease further processes the Pr55gag- and Pr55gag-pol-precursor proteins into p17MA, p24CA, p7NC, p6LI and the reverse transcriptase and integrase, respectively. The resulting particle undergoes structural changes forming the conical core structure typical for mature infectious HIV particles 
steps and to use them as a basis for the development of rational antiviral strategies.

\section{Viral proteins and amino acid sequences involved in morphogenesis}

All functions necessary and sufficient for HIV morphogenesis and particle release are localized in the sequence of the Pr55gag-precursor protein. This has been demonstrated by the fact that this protein is able to induce the formation of virus-like particles (VLPs) in the absence of further viral structural components like Pr160gag-pol and gp 120/gp41 proteins as well as regulatory polypeptides. When Pr55gag is transiently [43-45] or stably expressed in eucaryotic cells, or after infection of cells with recombinant vaccinia virus [46-48] or baculoviruses $[3,49$, 50 ], it promotes the formation and release of VLPs from the respective cells, which morphologically resemble noninfectious immature HIV particles. This noninfectious system allows the detailed analysis of regions within the Pr55gag proteins important for the formation of particulate structures.

Using these approaches large regions, particularly in the matrix protein $\mathrm{p} 17$, were identified to be essential for correct particle assembly. When residues 16 to 99 were deleted, the release of HIV particles from cells was reduced up to tenfold, budding events occurred preferentially at the membrane of the ER and incorporation of gp160 into the particulate structures was inhibited [24, 51]. Similar observations which defined regions of p17MA to be essential for glycoprotein incorporation were found by Yu and coworkers [25]. Furthermore, aminoterminal mutations were demonstrated to block intracellular transport of the gag-precursor protein [9]. The fact that regions spanning the cleavage site between p17MA and p24CA are also essential for the production of virus-like gag particles was shown using recombinant vaccinia viruses expressing altered gag proteins in which residues 99-154 (assembly important region 3, air3) were deleted [52]. In addition distinct other deletions within the p24CA (amino acids $211-241$ ) and the p6LI domain (436-471) of Pr55gag protein were without influence on the production of virus-like gag particles (Fig. 1). Furthermore, in terms of future vaccine development the latter two region could easily be substituted by peptide sequences from HIV envelope- and nef proteins to allow presentations of immunogenic epitopes by chimeric VLPs to the immune system [50, 52, 53].

More defined alterations in the sequence of the Pr55gag polyprotein exerting an influence on the production of virus-like gag particles were identified by von Poblotzki et al. [54] using recombinant vaccinia viruses with a deletion of 12 amino acids $(341-352)$ in the $\mathrm{p} 24 \mathrm{CA}$ protein (air2). In addition to this deletion, mutants were created by site-directed mutagenesis in this region by altering three consecutive amino acids to alanines (Table 1). According to these experiments, the functionally active residues localized in this domain could be defined as ATLEEM $(341-346)$ and CQG (350-352). This domain is of special interest since these amino acid sequences are highly conserved between the members of the family of mammalian immunodeficiency viruses. In different types of retroviruses, for example in Rous sarcoma virus (RSV), the carboxy-terminal domain of the capsid protein is important during the assembly process [55]. It is supposed to be involved in protein-protein interactions between individual gag-precursor molecules. It is noteworthy to mention that synthetic peptides spanning residues 339 - 350 inhibit 
the release of infectious HIV when added to cell cultures infected with the virus [56]. This aspect will be described in detail below.

When Pr55gag polyproteins containing the above-mentioned mutations were expressed by recombinant baculoviruses a significant reduction in the release of virus-like particles in comparison to wild-type HIV gag protein could be shown (Table 1). In contrast to the system of recombinant vaccinia viruses, certain numbers of particulate structures are still formed and released from the cell, probably due to the extremely high expression of Pr55gag under control of the baculovirusspecific polyhedrin promotor. Besides their reduction in number some mutant particles show a different, slightly higher, density in sucrose gradients when compared to the wild-type gag protein.

Similar effects were also shown when amino acids 47-59 in the p17 matrix protein (airl) were exchanged in tripletts to alanine residues. Expression of these constructs in recombinant baculoviruses likewise led to a reduced particle release, with partly altered densities (Table 1). In particular the region between amino acids 55 and 60 (EGCRQ) seems to be involved in the viral assembly process, since mutations at this site resulted in a strong inhibitory impact on particle release (Kattenbeck et al., unpublished data). In accord, proviral constructs with mutations in this region were recently shown to be replication defective $[24,25]$. This result is of special importance since synthetic peptides derived from this domain could also be shown to inhibit the production of infectious HIV [56].

\section{Possibilities to inhibit particle formation}

\section{Inhibitors of the viral protease}

As described above the endoproteolytic activity of the HIV-encoded protease is highly important for the process of virus maturation. When this enzyme becomes inactivated or is inhibited by certain drugs, the Pr55gag- and Pr160gag-pol-precursor proteins are not processed and the viral enzymes necessary for reverse transcription and integration of the viral genome in newly infected cells are not activated. The elucidation of the molecular structure of the HIV protease by X-ray crystallography [32] and molecular dynamic simulation [57] facilitated the development of improved compounds able to inhibit its proteolytic activity and, therefore, the succeeding maturation events. HIV-infected cells treated with inhibitors of the viral protease do not lead to the production of infectious viral particles [58, 59]. The particulate structures still budding from the cell surface represent immature, spherical HIV particles lacking the ability to form the conical capsid-core structure characteristic for infectious virions. Therefore, many investigations focused on the development of therapeutically active inhibitors of the HIV protease which may be used to suppress virus replication in HIV-infected individuals and limit the virus load in these persons. In addition to some approaches to disturb dimerization of the protease [60] or block its active site with various chemicals, synthetic peptides representing potential cleavage sites were used to inhibit the activity of the enzyme. This approach resulted in the development of R31-8959, one example of specific inhibitors of HIV protease, which has been administered to humans alone or in combination with inhibitors of reverse transcriptase [61, 62].

A general problem arising from inhibition of viral enzyme activity is the tendency of HIV to high mutability and the generation of drug-resistent virus strains. 


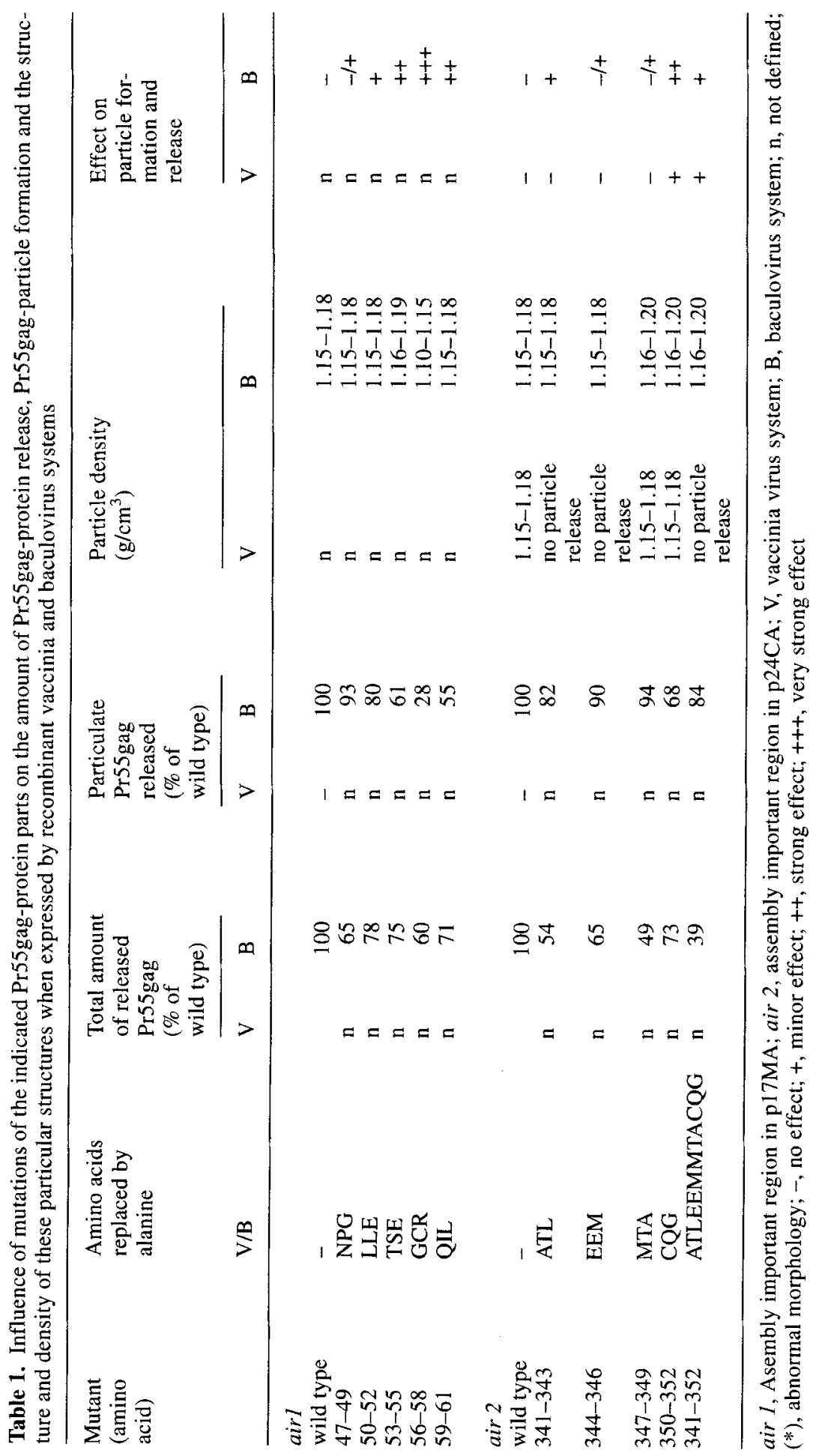


These mutated HIV variants can be easily isolated from HIV patients treated for therapeutic reasons with inhibitors either specific for virus protease or reverse transcriptase [63]. Therefore, it may prove to be more successful to disturb other nonenzymatic steps, like HIV-particle production, which do not suffer from mutational escape events. These functions may be highly conserved since they occur similarly in all budding lentiviruses, can be correlated with distinct protein domains and require a similar set of cellular and viral components.

\section{Compounds interfering with virus assembly}

When overlapping synthetic peptides - 22-24 amino acids in length -, which together represent the entire sequence of Pr55gag [64], were tested for their respective capacity to influence the assembly process of viral gag proteins by defining their ability to inhibit the release of infectious virus particles, four peptides were found which significantly reduced the amount of infectious HIV produced in the peptide-treated cell cultures. Two peptides represent overlapping compounds in the central part of $\mathrm{p} 17$, the other two peptides comprised sequences of the $\mathrm{p} 24$ capsid protein (Fig. 3, Table 2). The amount of released progeny virus was quantified indirectly by determining the activity of the HIV-specific reverse transcriptase in cell culture supernatants, hence reflecting the concentration of infectious virus in biological samples [65]. The level of infectious HIV was reduced about 100 -fold in cultures treated with the reactive peptides in comparison to the non-treated HIVinfected sample. The peptide effects were dose dependant in a range from 40 to $200 \mu \mathrm{g} / \mathrm{ml}$. Amounts of $25 \mu \mathrm{g} / \mathrm{ml}$ and below were not active. Furthermore, the effect could not be overcome by infecting cell cultures with an unnaturally high HIV concentration of up to $400 \mathrm{TCID}_{50}$. Similar effects could be observed on HIV release from chronically infected $\mathrm{H} 9$ cells and on acutely HIV-infected Jurkat cells. In the latter case, peptides were added to the cell cultures after virus adsorption. In both systems a similar reduction of infectious HIV particles released from the cells into the supernatant was found. When used in the indicated amounts, the peptides did not show toxic effects on the cells $[56,66]$. By use of shortened peptides spanning the overlapping parts of the active regions, we were able to define residues 47-59 (air1, NPGLLETSEGCRQ) in p17MA and 339-349 (air2, PAATLEEMMTA) in p24CA as active parts. To further characterize the functionally active amino acids, peptide derivatives in which tripletts of consecutive amino acids were substituted for alanine were synthesized and tested [56]. This allowed further delineation of the active regions to residues 50-58 (LLETSEGCR) in airl and 342-349 (TLEEMMTA) in air2.

When peptide-treated cells were analyzed in the electron microscope, particulate structures were found to be released from the cell surface which did not contain the conical core structure typical for mature lentivirus particles (Fig. 4). For this reason the vesicles were rather similar to immature virus particles. These observations indicated that the peptides exerted their activities by influencing a rather late step during HIV replication, namely virus assembly, since the early regulatory functions necessary for the synthesis of structural proteins were obviously not af fected. Furthermore the ability of the peptide-treated cells to form syncytia in the cell cultures showed that the synthesis and incorporation of gp120/gp41 into the cell membrane was not negatively influenced. To test what amounts of the peptides might be cell associated and what proportion of the added molecules may 


\title{
Inhibition of HIV production
}

\author{
by gag peptides
}

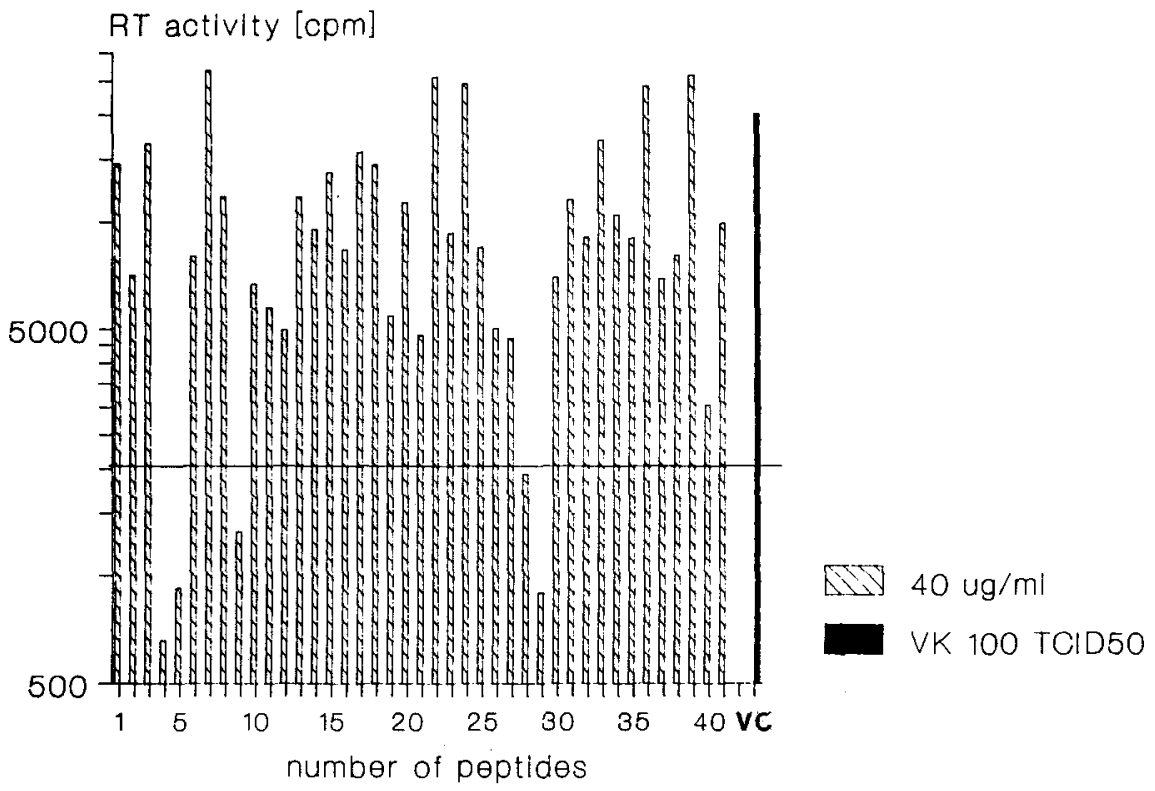

Fig. 3. Influence of the 41 Pr55gag protein-derived peptides on the release of infectious HIV particles from infected Jurkat cells. Cells were treated with $40 \mu \mathrm{g} / \mathrm{ml}$ peptide after HIV adsorption. After 3 days the supernatants were analyzed for the presence and the amount of infectious HIV using aliquots of $100 \mu 1$ to infect fresh Jurkat cells which were incubated for 2 weeks. The amount of HIV in the supernatants of these indicator cells was determined by quantification of reverse transcriptase activity. $V C$ Positive control; HIV-infected Jurkat cells without peptides, the negative control is given by the value of reverse transcriptase activity in the supernatants of uninfected cells

pass the lipid bilayer, tyrosinylated forms of the shortened variants of the active compounds were synthesized and labelled with ${ }^{125} \mathrm{I}$. The radioactively labelled peptides were incubated in various concentrations with uninfected Jurkat cells. In the case of the peptide derived from airl, $21 \%$ remained cell associated after several washing procedures. For the air 2 peptide an amount of $6.5 \%$ was shown to be irremoveable (Table 3 ). The percentage of radioactivity associated with the cell was not altered to any major extent using either different amounts of labelled peptides in concentrations below saturation or by altering the incubation time from 2 to $4 \mathrm{~h}$. These observations indicated that defined quantities of the peptides are, at least, cell associated, i.e., taken up by the cell. Therefore, we postulate that the peptides pass the cellular membrane by still unknown mechanisms. Peptide transporters have been described to be present in the membrane of the ER. They were shown to translocate proteolytically degraded peptides into this compartment where they associated with major histocompatibility complex (MHC) class I proteins. Recently, peptide-transporter complexes also have been found in the membranes of rabbit small intestine and various other cells [67]. It may be assumed that similar proteins, which actively transport organic compounds, are involved in the 


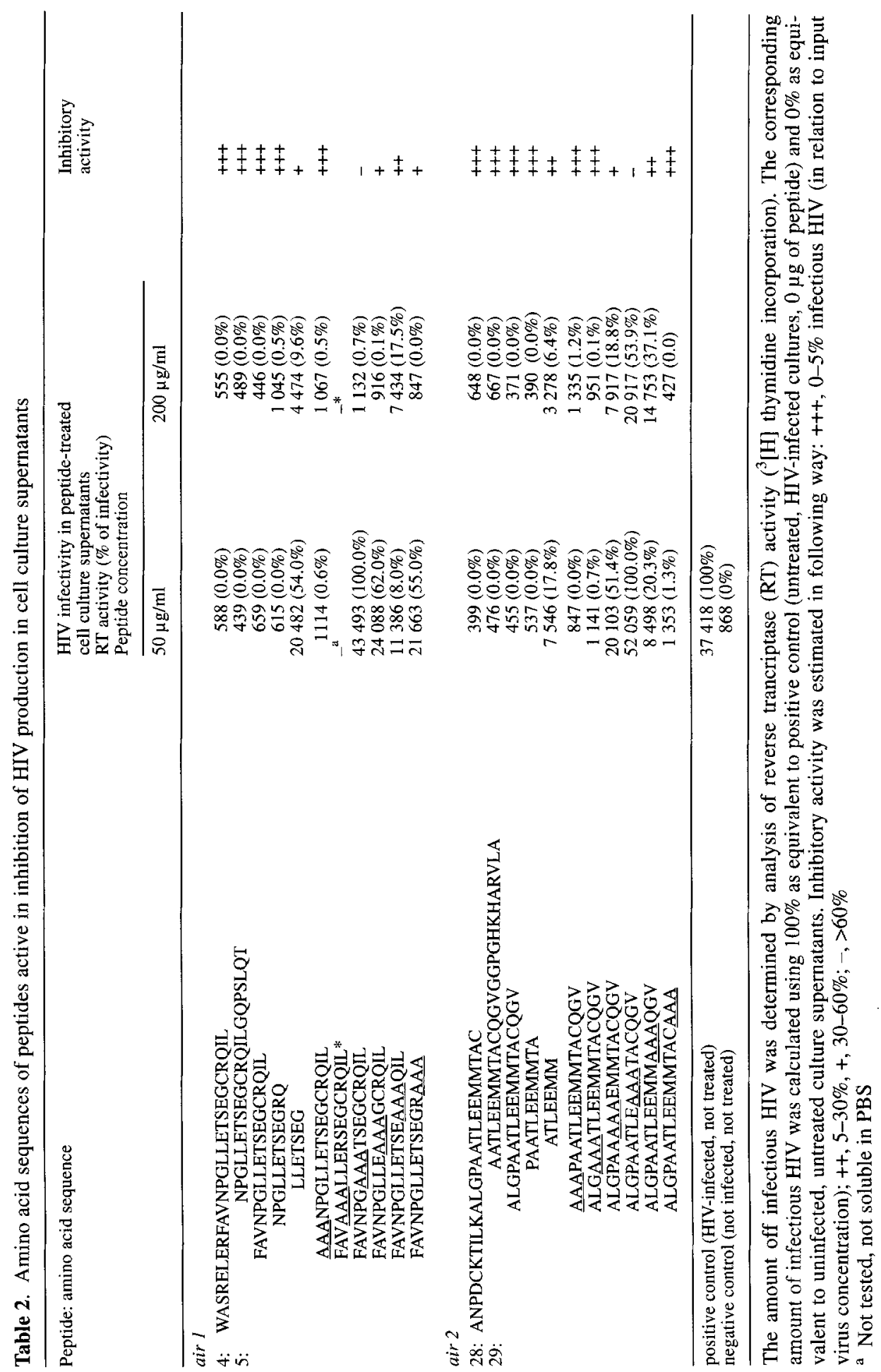



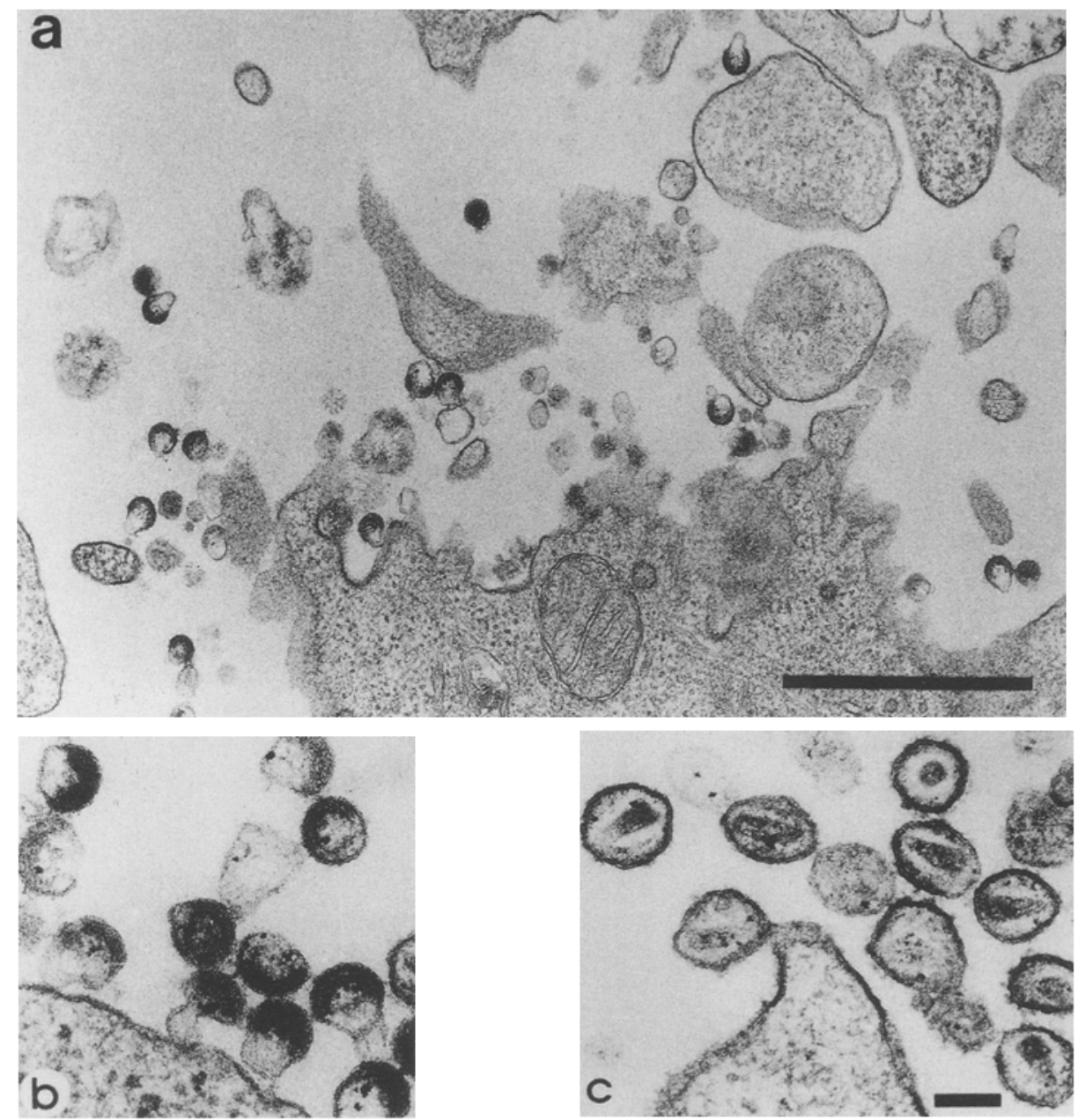

Fig. 4. A Electron microscopic presentation of the particulate structures released from peptide air2-treated HIV-1-infected H9 cells; only spherical particles with characteristics of immature HIV are released from the cell surface. Bar represent $1 \mu \mathrm{m}$. B Magnification of immature particles budding from HIV-infected peptide-treated H9 cells. C Positive control of HIV-infected, non-peptide-treated cells: Mature HIV particles with conical capsid structures are released from H9-cells. Bar represents $100 \mathrm{~nm}$

import of the peptides into the cytoplasm of the cell where they may exert their respective functions.

The particulate structures released from peptide-treated cells were similar to immature particles produced in HIV-infected cell cultures treated with inhibitors specific for the HIV protease [58], or when the sequences encoding the protease were mutated [68]. This morphological similarity implied that the active peptides could represent unnatural substrates exerting their inhibitory activity in a competitive way to the cleavage sites in the Pr55gag- and Pr160gag-pol-precursor proteins. To test this possibility, the peptides were tested in an in vitro system for their 
Table 3. Amino acid sequences active in form of synthetic peptides to inhibit the release of infectious HIV from cell cultures.

\begin{tabular}{ll}
\hline Peptide/amino acid sequence & Protease Cellular \\
& inhibition uptake \\
& IC $50(\mu \mathrm{g} / \mathrm{ml}) \%$ \\
\hline
\end{tabular}

air 1

4: WASRELERFANGPLLETSEGCRQIL

5: $\quad$ NPGLLETSEGCRQILGQLQPSLQT

NPGLLETSEGCRQIL

59.0

530.0

$18.5 \quad 21$

air2

28: ANPDCKTILKALGPAATLEEMMTAC

$>666.0$

29:

AATLEEMMTACQGVGGPGHKARVLA $\quad 240.0$

AATLEEMMTAC $>666.0$

6.5

The respective influence of the peptides on protease activity and the percentage of iodinated peptides that remain associated with the cells after incubation periods are indicated

respective activities to inhibit the HIV-1-specific protease. The majority of the derivates had no inhibitory influence on this enzyme and particularly the shortened forms of the active peptides did not show any activity at all (Table 3 ). Therefore, the inhibitory effect on particle release appeared not to be due to an effect on protease activity.

Besides viral protease, other viral or cellular functions might also be the target for the peptides. One of the cellular processes involved might be at the level of enzymes catalyzing protein folding. It was reported [10] that cyclophilin, representing a member of the family of cellular chaperones, binds to the gag-precursor proteins and might influence the correct assembly of HIV components. The regions of Pr55gag proposed to be involved in the binding of cyclophilin do not correlate with the domains air 1 and air 2 from which the active peptides were derived. Nevertheless, we can not exclude that the peptides might bind to cyclophilin and influence its catalytic function. For this reason we analyzed the peptides for their respective capacity to bind or inhibit the function of cyclophilin. However, none of the peptides active in the inhibition of release of infectious HIV bound or influenced the activity of cyclophilin. Hence, we conclude that this cellular enzyme like the viral protease - is not a target for the peptides.

At the moment the exact mechanism of activity of the peptides remains unresolved. Various cellular systems involved in modification and transport of the Pr55gag-precursor protein may be involved including further cellular chaperones, since so far only the interaction with cyclophilin A and B has been tested. It also remains possible that the peptides may exert their inhibitory activity on the release of infectious particles by direct interaction with the Pr55gag-precursor proteins incorporated in the cytoplasmic membrane, thus reducing the concentration of these polypeptides. Incorrect budding, in combination with incomplete interaction of individual gag molecules, and consequent activation of the HIV-specific protease, may lead to the formation of immature noninfectious particles. It is important to note that the inhibitory effect can not be washed out by diluting the immature particles in fresh medium or buffers. Even then the particulate structures remain noninfectious. This observation is in contrast to the activities of inhibitors of viral enzymes such as protease or reverse transcriptase. 
The fact that both synthetic peptides derived from Pr55gag-protein sequences influence virus morphogenesis and the identical original amino acid regions are important for particle formation led us to the conclusion that intrinsic and defined functions of the gag protein are affected $[24,25,54]$. When certain peptides were added to HIV-infected cell cultures, the overall formation of virus particles was reduced and the shape of the released particles was altered. If functionally important residues of these active synthetic peptide derivatives were deleted or exchanged, their inhibitory action on particle release ceased. When residues of the same region in the Pr55gag sequence were exchanged in gag protein expression systems, the production of HIV-like gag particles was completely suppressed or altered. This occurs because deletion or alteration of these functionally active residues at the DNA level affects every Pr55gag molecule. In contrast, when synthetic peptides are added to cells, which still contain functionally active HIV-gag-precursor proteins due to the viral infection, peptides can only disturb but not block HIV assembly. Here, the effect may depend on the concentration of and the quantitative relation between the synthetic peptides and the viral precursor proteins and, hence, the amount of HIV produced in individual cells.

Recent, unpublished results further support our initial observations ( $S$. Modrow et al., unpublished). It could be shown that the HIV-1-specific gag protein-derived peptides also inhibit the release of HIV-2 infectious particles and peptides derived from the respective gag-protein sequences of simian immunodeficiency virus (SIV; p18MA: FGLAESLLENKEGCQKILS; p27CA: GLGTNPTLEEMLTACQG) significantly reduced the release of SIV gag proteins from infected cells (Table 4). In particular, the investigated region of the capsid protein seems to be essential for particle formation. The amino acid sequences here are highly conserved among HIV, SIV and feline immunodeficiency virus and can neither be altered in the synthetic peptides without losing inhibitory activity nor in recombinant Pr55gag without losing the capacity to form virus-like particles (Fig. 5). These findings reflect the fact that the observed inhibitory effect of the synthetic peptides is not virus-

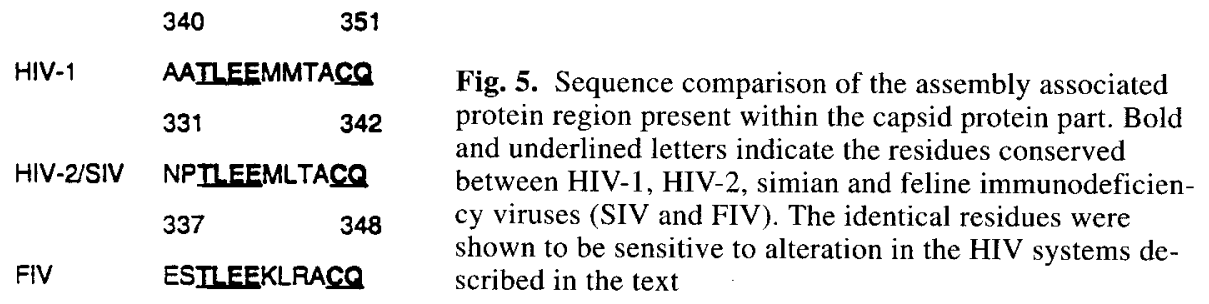

Table 4. Influence of SIV gag protein-derived peptides corresponding to the regions active in inhibition of HIV-1-particle release on the release of SIV (strain J5) from infected cells measured by the quantification of SIV-gag-proteins p27 in capture assays after 6 days of incubation

Peptide sequence $\quad$ SIV p27 capsid protein cell culture supernatants $(\mathrm{ng} / \mathrm{ml})$ (\% inhibition in relation to $\mathrm{p} 27$ production of controls)

$\begin{array}{ll}\text { FGLAESLLENKEGCQKLIS } & 1560(84 \%) \\ \text { GLGTNPTLEEMLTACQG } & 1320(86 \%) \\ \text { Virus control } & 9600(0 \%)\end{array}$


type specific. These data indicate that inhibition of virus assembly by synthetic peptides or derivatives thereof might open new perspectives for antiviral treatment by affecting lentivirus particle assembly.

\section{Conclusions and summary}

Using two different experimental systems we were able to define two distinct regions in the sequence of gag proteins of HIV which function in the assembly of structural components of the virus and particle morphogenesis. Since assembly could be inhibited by addition of synthetic non-toxic peptide compounds, a new group of antiviral agents may be developed. This approach represents a completely new inhibitory principle. Besides irreversibly blocking the synthesis of progeny virus, this class of peptidomimetic inhibitors might be of special interest since noninfectious particles are released which should still be capable of stimulating the immune system and helping to induce an improved immune status. Those effects have been observed when mice were vaccinated with Rauscher murine leukemia virus and treated simultaneously with zidovudine and interferon- $\alpha$ [69].

Besides the development of antiviral compounds active viral protein regions actively involved in morphogenesis may be developed for applications in gene therapy using assembly defective gag proteins to negatively influence virus production via transdominant-negative effects.

Acknowledgements. The authors thank H. R. Gelderblom, Robert-Koch Institut, Berlin, Germany and G. Wanner, Ludwig-Maximilians-Universität, München, Germany, for electron microscopy, A. Brunner for assistance in peptide synthesis, J. Stott and T. Corcoran, NIBSC, Hertfordshire, UK, for testing SIV gag protein-derived peptides in SIV systems, J. Buchner and H. Lilie, Universität Regensburg for testing peptide activity on cyclophilin. We thank D. J. Schendel, Ludwig-Maximilians-Universität, München for critical reading of the manuscript. Parts of this work were supported by the Bundesgesundheitsamt, Forschungsverbund BGA $1 / 88$, project B5 and Wilhelm Sander Stiftung, grant 92.014.1 to S. M.

\section{References}

1. Barré-Sinoussi F, Cherman JC, Rey F (1983) Isolation of a T-lymphocyte retrovirus from a patient with risk for acquired immunodeficiency syndrome. Science 220: $868-871$

2. Popovic M, Sarngadharan MG, Reed E, Gallo RC (1984) A method for detection, isolation and continuous production of cytopathic human T-lymphotropic retroviruses of the HTLV family (HTLV-III) from patients with AIDS and pre-AIDS. Science 224: 497-500

3. Gheysen D, Jacobs E, de Forestra F, Thiriart C, Francotte M, Thine SD, de Wilde M (1989) Assembly and release of HIV-1 precursor Pr55gag virus-like particles from recombinant baculovirus-infected insect cells. Cell 59:103-112

4. Gelderblom HR, Özel M, Pauli G (1989) Morphogenesis and morphology of HIV: structurefunction relations Arch Virol 106:1-13

5. Henderson LE, Bowers MA, Sowder RC, Serabyn SA, Johnson DG, Bess JW, Arthur LO, Bryant D, Fenselen D (1992) Gag-proteins of the highly replicative MN strain of human immunodeficiency virus type 1: postranslational modifications, proteolytical processings and complete amino acid sequences. J Virol 66: 1856-1865

6. Henderson LE, Sowder RC, Copeland TD, Oroszlan S, Beneveniste RE (1990) Gag precursors of HIV and SIV are cleaved into six proteins found in the mature virious. J Med Primatol 19:411-419

7. Veronese FD, Copeland TD, Oroszlan S, Gallo RC, Sarngadharan MG (1988) Biochemical and immunological analysis of human immunodeficiency virus gag-gene products $\mathrm{p} 17$ and p24. J Virol 62:795-801 
8. Göttlinger HG, Sodroski JG, Haseltine WA (1989) Role of capsid precursor processing and myristoylation in morphogenesis and infectivity of human immunodeficiency virus type 1 . Proc Natl Acad Sci USA 86:5781-5785

9. Yuan X, Yu X, Lee TH, Essex M (1993) Mutations in the N-terminal region of human immunodeficiency type 1 matrix protein blocks intracellular transport of the gag-precursor. $\mathrm{J}$ Virol 67:6387-6394

10. Luban J, Bossolt KL, Franke EK, Kalpana GV, Goff SP (1993) Human immunodeficiency virus type 1 gag-protein binds to cyclophillins A and B. Cell 73: 1067-1078

11. Jacks T, Power M, Masiarz FR, Luciv PA, Barr PJ, Varmus HE (1988) Characterization of ribosomal frameshifting in HIV-1 gag-pol expression. Nature 331:280-283

12. Wilson W, Braddock M, Adams SE, Rathja PD, Kingsman SM, Kingsman AJ (1988) HIV expression strategies: ribosomal frameshifting is directed by a short sequence both in mammalian and yeast systems. Cell 55:1159-1169

13. Walter P, Gilmore R, Blobel G (1984) Protein translation across the endoplasmatic reticulum. Cell 38:5-8

14. Kosarsky K, Penman M, Basiripour L, Haseltine W, Sodroski J, Krieger M (1989) Glycosylation and processing of the human immunodeficiency virus type 1 envelope protein. $\mathbf{J}$ AIDS 2: $163-169$

15. Ranajit P, Hoke GM, Sarngadharan MG (1989) Role of oligosaccharides in the processing and maturation of envelope glycoproteins of HIV-1. Proc Natl Acad Sci USA 86:33843388

16. Dewar RL, Vasudevachari MB, Natarajan V, Salzman NP (1989) Biosynthesis and processing of human immunodeficiency virus type 1 envelope glycoproteins: effect of monensin on glycosylation and transport. J Virol 63:2452-2456

17. Willey RL, Bonifacino JS, Potts BJ, Martin MA, Klausner RD (1988) Biosynthesis, cleavage and degradation of the human immunodeficiency virus type $1 \mathrm{gp} 160$. Proc Natl Acad Sci USA 85:9580-9584

18. Hallenberger S, Bosch V, Angliker H, Shaw E, Klenk HD, Gartem W (1992) Inhibition of furin-mediated cleavage activation of HIV-1 glycoprotein 160. Nature 360:385-391

19. Aldorini A, Young RA (1990) Mutations of RNA and protein sequences involved in HIV-1 packaging result in production of non-infectious virus. J Virol 64: 1920-1926

20. Gorelik RJ, Nigida SM, Bess JW, Arthur LO, Henderson LE, Rein A (1990) Non-infectious human immunodeficiency virus type 1 mutants deficient in genomic RNA. J Virol 64: $3207-3211$

21. South TL, Blake PR, Hare DR, Summers MF (1991) C-terminal retroviral-type Zink finger domain from HIV-1 nucleocapsid protein structurally similar to the $\mathrm{N}$-terminal Zink finger domain. Biochemistry 30:6342-6349

22. Danull J, Surovoy A, Jung G, Moelling K (1994) Specific binding of HIV-1 nucleocapsid protein to PSI RNA in vitro requires $\mathrm{N}$-terminal zinc finger and flanking basic amino acid residues. EMBO J 13:1525-1533

23. Dorfman T, Luban J, Goff SP, Haseltine WA, Göttlinger HG (1993) Mapping of functionally important residues of a cysteine-histidine box in the human immunodeficiency virus type 1 nucleocapsid protein. J Virol 67:6159-6169

24. Dorfman T, Mammano F, Haseltine WA, Göttlinger HG (1994) Role of the matrix protein in the virion association of the human immunodeficiency virus type 1 envelope glycoprotein. J Virol 68: 1689- I696

25. Yu X, Yuan X, Matsuda Z, Lee T-H, Essex M (1992) The matrix protein of human immunodeficiency virus type 1 is required for incorporation of viral envelope proteins into mature virus. J Virol 66:4966-4971

26. Gelderblom HR, Haussmann EHS, Özel M, Pauli G, Koch MA (1987) Fine structure of human immunodeficiency virus (HIV) and immunolocalization of structural proteins. Virology 156: $171-176$

27. Gelderblom HR (1991) Assembly and morphology of HIV: potential effect of structure on viral function. AIDS 5:617-638

28. Lavalle C, Yao XJ, Ladha A, Göttlinger H, Haseltine WA, Cohen EA (1994) Requirement of the pr55gag precursor for incorporation of the vpr product into HIV type 1 viral particles. J Virol 68: 1926-1934 
29. Göttlinger HG, Dorfman T, Cohen E, Haseltine WA (1993) Vpu protein of human immunodeficiency virus type 1 enhances the release of capsids produced by gag gene constructs of widely divergent retroviruses. Proc Natl Acad Sci USA 90: 7381-7385

30. Paxton W, Connor RI, Landau NR (1993) Incorporation of vpr into human immunodeficiency virus type 1 virions: requirement for the $\mathbf{p} 6$ region of gag and mutational analysis. J Virol $67: 7229-7237$

31. Höglund S, Ohagen A, Lawrence K, Gabuzda D (1994) The role of vif during packing the core of HIV-1. Virology 201:349-355

32. Wlodawer A, Miller M, Jaskolski M, Sathyanarayana BK, Baldwin E, Weber ET, Selk LM, Clawson L, Schneider J, Kent SB (1989) Conserved folding in the retroviral proteases: crystal structure of a synthetic HIV-1 protease. Science 245:616-621

33. Oroszlan S, Luftig RB (1990) Retroviral proteinases. Curr Top Microbiol Immunol 157: $153-185$

34. Haseltine WA (1991) Molecular biology of the human immunodeficiency virus type 1. FASEB J 5:2349-2360

35. Nutt RF, Brady SF, Darke PL, Ciccarone TM, Colton CD, Nutt EM, Rodkey JA, Bennet CD, Waxman LH, Sigal IS (1988) Chemical synthesis and enzymatic activity of a 99 residue peptide with a sequence proposed for the HIV-1 protease. Proc Natl Acad Sci USA 85: 71297133

36. Oswald M, Helm K von der (1991) Fibronectin as a non-viral substrate for the HIV proteinase. FEBS Lett 292: 298-300

37. Wang CT, Barklis E (1993) Assembly, processing, and infectivity of human immunodeficiency virus type 1 mutants. J Virol 67:4264-4273

38. Tritch RJ, Cheng YSE, Yin HF, Erickson-Vitanen S (1991) Mutagenesis of protease sites in the human immunodeficiency virus type $1 \mathrm{gag}$ polyprotein. J Virol 65:922-930

39. Mervis RJ, Ahmad N, Lillehoj EP, Raum MG, Salazar FH, Chan HW, Venkatesan S (1988) The gag-gene products of HIV-1: alignment within the gag open reading frame, identification of posttranslational modifications and evidence for alternative gag precursors. J Virol 62: 3993-4002

40. Ehrlich LS, Agresta BE, Carter CA (1992) Assembly of recombinant HIV-1 capsid proteins in vitro. J Virol 66:4874-4883

41. Göttlinger HG, Dorfman T, Sodroski JG, Haseltine WA (1991) Effects of mutations affecting the p6 gag protein on human immunodeficiency virus particle release. Proc Natl Acad Sci USA 88: $3195-3199$

42. Höglund S, Öferstadt L-G, Nilson A, Özel M, Winkel T, Skoglund U, Gelderblom HR (1991) Analysis of the assembly of the HIV-core electron microscope tomography. In: Pearl LE (ed) Retroviral proteases control of maturation and morphogenesis. Macmillan Press, London, pp $149-157$

43. Smith AJ, Cho MJ, Hammarskjold ML, Rekosh D (1990) Human immunodeficiency virus type 1 pr55gag and pr160gag-pol expressed from a simian virus 40 late replacement vector are efficiently processed and assembled into virus like particles. J Virol 60:2743-2750

44. Mergener K, Fäcke M, Walter R, Brinkmann V, Gelderblom HR, Kräusslich GH (1992) Analysis of HIV particle formation using transient expression of subviral constructs in mammalian cells. Virology 186: $25-39$

45. Vernon SK, Murthy S, Wilhelm J, Chanda PK, Kalyan N, Lee S-G, Hung PP (1991) Ultrastructural characterization of human immunodeficiency virus type 1 gag-containing particles assembled in a recombinant adenovirus vectorsystem. J Gen Virol 72:12431251

46. Karacostes V, Nagashima K, Gonda MA, Moss B (1989) Human immunodeficiency viruslike particles produced by a vaccinia virus vector. Proc Natl Acad Sci USA 86:89648967

47. Haffar O, Ganigues J, Travis B, Moran P, Zarling J, Hu SL (1990) Human immunodeficiency virus-like nonreplicating particles assemble in a recombinant vaccinia virus expression system. J Virol 64:2653-2659

48. Wagner R, Fließbach H, Modrow S, Brunn A von, Wolf H, Böltz T, Gelderblom H (1991) Expression of autologous pr55 and pr55/gp 120-V3 core particles: a new approach to new vaccines including prevention of AIDS. In: Chanock RM et al. (eds) Vaccines 91 . Cold Spring Harbor Laboratory, Cold Spring Harbor, pp 109-114 
49. Royer M, Cerutti M, Gay B, Hong SS, Deranchelle G, Boulanger P (1991) Functional domains of HIV-1 gag-polyprotein expressed in baculovirus-infected cells. Virology 184: 417-422

50. Wagner R, Fließbach H, Wanner G, Motz M, Niedrig M, Deby G, Brunn A von, Wolf H (1992) Studies on processing, particle formation and immunogenicity of the HIV-1 gag gene product: a possible component of a HIV vaccine. Arch Virol 127:139-152

51. Fäcke M, Janetzko A, Shoeman RL, Kräusslich H-G (1993) A large deletion in the matrix domain of the human immunodeficiency virus gag gene redirects virus particle assembly from the plasma membrane to the endoplasmic reticulum. J Virol 67:4972-4980

52. Wagner R, Deml L, Fließbach H, Wanner G, Wolf H (1994) Assembly and extracellular release of chimeric HIV-1 pr55gag retrovirus-like particles. Virology 200: 162-175

53. Wagner R, Böltz T, Deml L, Modrow S, Wolf H (1993) Induction of cytolytic T-lymphocytes directed towards the V3 loop of the human immunodeficiency virus type 1 external glycoprotein gp120 by p55gag/V3 chimeric vaccinic viruses. J Gen Virol 74:1261-1269

54. Poblotzki A von, Wagner R, Niedrig M, Wanner G, Wolf H, Modrow S (1993) Identification of a region in the pr55gag-polyprotein essential for HIV-1 particle formation. Virology 193: $981-985$

55. Wills JW, Craven RC (1991) Form, function and use of retroviral gag proteins (editorial). AIDS 5:639-654

56. Niedrig M, Gelderblom HR, Pauli G, März J, Bickhard H, Wolf H, Modrow S (1994) Inhibition of infectious human immunodeficiency virus type 1 particle formation by gag protein-derived peptides. J Gen Virol 75: 1469-1474

57. Navia MA, Fitzgerald PM, McKeever BM, Leu CT, Heimbach JC, Herber WK, Sigal JS, Darke PL, Springer JP (1989) Three-dimensional structure of aspartyl protease from human immunodeficiency virus type 1 . Nature 337:615-620

58. Schätzl H, Gelderblom HR, Nitschko H, Helm K von der (1991) Analysis of non-infectious HIV particles produced in the presence of HIV proteinase inhibitors. Arch Virol 5:233-239

59. Kaplan AH, Zack JA, Knigge M, Paul DA, Kempf DJ, Norbeck DW, Swanstrom R (1993) Partial inhibition of the human immunodeficiency type 1 protease results in aberrant virus assembly and the formation of noninfectious particles. J Virol 67:4050-4055

60. Babe LM, Rose J, Craik CS (1992) Synthetic "interface" peptides alter dimeric assembly of the HIV-1 and 2 proteases. Protein Sci 1: $1244-1253$

61. Johnson VA, Merril DP, Chou TC, Hirsch MS (1992) Human immunodeficiency type 1 (HIV-1) inhibitory interactions between protease inhibitor RO 31-8959 and zidovudine, $2^{\prime}, 3$ dideoxycytidine, or recombinant interferon alpha $\mathrm{A}$ against zidovudine-sensitive or -resistant HIV-1 in vitro. J Infect Dis 166:1143-1146

62. Fischl MA (1994) Combination antiretroviral therapy for HIV-infection. Hosp Pract (Off Ed) 29:43-48

63. Boucher CA, O'Sullivan E, Mulder JW, Ramautarsing C, Kellam P, Darby G, Lange M, Goudmit J, Larder BA (1992) Ordered appearance of zidovudine resistance mutations during treatment of 18 human immunodeficiency virus-positive subjects. J Infect Dis 165: 105-110

64. Haist S, März J, Wolf H, Modrow S (1992) Reactivities of HIV-1 gag-derived peptides with antibodies of HIV-1 infected and uninfected humans. AIDS Res Hum Retroviruses 8: $1909-1917$

65. Land S, Beaton F, McPhee DA, Gust ID (1989) Comparison of the antigen (p24) assay and reverse transcriptase activity for detection of human immunodeficiency type 1 replication. J Clin Microbiol 27:486-489

66. Niedrig M, Bickhard H, Gelderblom HR, Pauli G, Modrow S, Poblotzki A von, Wagner R, Wolf H (1993) Assembly of HIV-gag proteins: a target for antiviral therapy? In: Ginsberg HS et al. (eds) Vaccines 93, Cold Spring Harbor Press, Cold Spring Harbor, pp 209-214

67. Fei Y-J, Kanai Y, Nussberger S, Ganapathy V, Leibach FH, Romero MF, Singh SK, Boron WF, Hediger MA (1994) Expression cloning of a mammalian proton-coupled oligopeptide transporter. Nature 368:536-566

68. Park J, Morrow CD (1993) Mutations in the protease gene of human immunodeficiency virus type 1 affect release and stability of virus particles. Virology 194: 843-850

69. Ruprecht RM, Mullaney S, Bernard LD, Gama Sosa MA, Hom RC, Finberg RW (1990) Vaccination with a live retrovirus: the nature of the protective immune response. Proc Natl Acad Sci USA 87:5558-5562 